See discussions, stats, and author profiles for this publication at:

http://www.researchgate.net/publication/259510236

\title{
Overconfidence bias and conjunction fallacy in predicting outcomes of football matches
}

ARTICLE in JOURNAL OF ECONOMIC PSYCHOLOGY · JANUARY 2013

Impact Factor: 1.21 · DOI: 10.1016/j.joep.2013.12.003

DOWNLOADS

445

2 AUTHORS:

Nikola Erceg

University of Zagreb

1 PUBLICATION 0 CITATIONS

SEE PROFILE
VIEWS

1,685
Zvonimir Galic

University of Zagreb

16 PUBLICATIONS 22 CITATIONS

SEE PROFILE 


\title{
Overconfidence bias and conjunction fallacy in predicting outcomes of football matches
}

\author{
Nikola Erceg*, Zvonimir Galić \\ University of Zagreb, Croatia
}

\section{A R T I C L E I N F O}

\section{Article history:}

Received 7 November 2012

Received in revised form 25 November 2013

Accepted 13 December 2013

Available online $\mathrm{xxxx}$

\section{JEL classifications:}

C93

D12

D80

PsycINFO classification:

2300

3900

\section{Keywords:}

Overconfidence bias

Conjunction fallacy

Betting

Probability

Frequency

\begin{abstract}
A B S T R A C T
The aim of this study was to explore the occurrence of the overconfidence bias and the conjunction fallacy in betting behavior among frequent and sporadic bettors and to test whether it was influenced by the task format (probability vs. frequencies). Frequent bettors $(N=67)$ and sporadic bettors $(N=63)$ estimated whether the bets on football games presented to them via an on-line questionnaire would be successful. The bets consisted of singles (one match outcomes) and conjunctions (two matches outcomes), and were presented either in probability or frequency terms. Both frequent and sporadic bettors showed similar levels of the overconfidence bias. However, the frequent bettors made the conjunction fallacy more often than the sporadic bettors. The presentation of the task in the frequency terms significantly reduced the overconfidence bias in comparison to the evaluations in probability terms, but left the conjunction fallacy unaffected.
\end{abstract}

(c) 2013 Elsevier B.V. All rights reserved.

\section{Introduction}

In addition to thrill, excitement and the possibility of a financial gain as the most obvious reasons that "drive" people into betting, it is possible that certain irrational beliefs and cognitive biases play a significant role in this type of behavior. For example, cognitive biases may lead to overconfidence regarding the possibility of winning and, consequently, wrong decisions about money investments (Nilsson \& Andersson, 2010). In this paper we described the results of a study that investigated the occurrence of the two cognitive biases, the overconfidence bias and the conjunction fallacy, in predictions of outcomes of football matches. Specifically, we examined the differences in susceptibility to the two biases between people who frequently bet on sport outcomes (frequent bettors) and people who rarely or never bet on sport outcomes (sporadic bettors), and explored whether those biases can be reduced by the task format. Previous studies have shown that formulating the problem in terms of frequency rather than probability could help decrease the biases (Hertwig \& Gigerenzer, 1999; Kahneman \& Tversky, 1996). To the best of our knowledge, our study represents the first attempt of exploring problem

\footnotetext{
* Corresponding author. Address: Department of Psychology, University of Zagreb, Luciceva 3, 10000 Zagreb, Croatia. Tel.: +385 992023004.

E-mail address: i.nikola.erceg@gmail.com (N. Erceg).
} 
formulations in terms of frequencies in the field of sports betting. Before describing our study in more detail, we will review the literature on the overconfidence bias, the conjunction fallacy, and the influence of task format on cognitive biases.

\subsection{Overconfidence bias}

Many studies have indicated that the phenomenon of overconfidence in judgments and decisions is widespread and frequent in occurrence. It has been identified in everyday life activities such as the opinion about one's own driving capabilities (Svenson, 1981) and evaluations of various future outcomes (e.g., academic, social and recreational choices for the following year, Vallone, Griffin, Lin, \& Ross, 1990). More importantly the overconfidence in decisions has been regularly observed in the work of many professionals such as doctors, lawyers, engineers, psychologists or security analysts (see Belsky \& Gilovich, 1999, for a review). In its essence the overconfidence bias reflects the fact that the confidence that people have in their judgments does not match real accuracy of those judgments. Montier (2007) reported the results of a study in which doctors, while claiming that they were $90 \%$ confident about their diagnoses, were actually accurate in less than $15 \%$ of cases. Torngren and Montgomery (2004) showed that when given a task to select the stock that would achieve the highest price growth in the following 30 days, the laymen were 59\% confident about their choices, whereas the experts reported confidence at $65 \%$ level. The actual success of both groups was below chance - they would have made better choices if they followed a randomized procedure. Interestingly, the experts who claimed that they were $100 \%$ confident about their choice of stock were correct only $15 \%$ of the time. In a well-known paper about the relationship between intuition and expertise, Klein and Kahneman (2009) concluded that whenever the predictability of the environment in which the judgment is made is questionable and the possibility of learning regularities of that environment is small, even experts do not make accurate judgments. The prevalence of the overconfidence bias and dangerous consequences that could follow from it make this bias an important object of scientific research.

There are two predominant paradigms in studies of overconfidence: confidence in binary decisions and interval prediction format. The classic way of studying overconfidence is the one where researchers provide a series of questions for which two alternative answers are given, and the respondents' task is to pick the correct one. This type of exploring the overconfidence bias has two varieties. With the half-range format, questions take the following form: "Who lived longer - Ho Chi Minh or Claude Monet?" Respondents select the answer they think is more likely to be correct and express their confidence on a probability scale ranging from .50 to 1.0. With the full-range format, respondents receive the statement "Ho Chi Minh lived longer than Claude Monet did." (or vice versa) and give the probability that the statement is true on a scale from 0 to 1 (Klayman, Soll, Juslin, \& Winman, 2006). The third way of expressing confidence is interval production format where participants are asked to specify the so-called "confidence interval" in their decision (Alpert \& Raiffa, 1982). This interval represents the range of values within which the correct answer is located, depending how confident a subject is about the answer (for example, I am 90\% certain that the city of Zagreb, Croatia has a population between 700,000 and 1,000,000 inhabitants). Although confidence in a knowledge domain varied depending on the assessment format (Hansson, 2007), all three research paradigms revealed that individuals overestimate accuracy of their judgments.

One of the possible explanations of the overconfidence bias is related to the mechanism through which individuals integrate evidences related to the decision. According to Griffin and Tversky (1992), evaluation of confidence about a certain hypothesis requires an integration of various types of evidence. In most problems it is possible to distinguish between two dimensions of evidence: the strength or the extremity of the evidence and the weight or the predictive validity of the evidence. The interplay of the two dimensions of evidence determines the level of confidence in a decision. Although predictive validity reflects the probability of an event occurrence, overconfidence is often based on the strength of the evidence. The combination of these factors explains why experts are often overconfident in their decisions. In situations where predictability is low every additional quantity of experts' knowledge increases the strength of evidence, but has no effect on its weight (i.e., predictive validity). In these situations experts are much more confident in their judgment, but the unpredictability of the situation disables the reflection of experience in the decision accuracy.

Few studies that dealt with the overconfidence bias in sport betting mostly confirmed the assumption that experts are overconfident in their judgments, and that their overconfidence stems from reliance on wrong types of evidence. Andersson, Ekman, and Edman (2003) showed that sport journalists, soccer fans and coaches ("experts") were no more successful than Swedish and American students with no knowledge about football ("laymen") in their predictions of which teams will win the second round of FIFA World Cup 2002. However, they were considerably more confident in their evaluations. The accuracy in both groups was at the level of random guessing, but while "laymen" admitted that their predictions were based on mere recognition of teams, the "experts" claimed that they relied on a wide search and an analysis of available information. Similar results were reported by Andersson, Memmert, and Popowicz (2009). When predicting which teams will qualify for the second round of the FIFA World Cup 2006 tournament, the "experts" were no more successful than the "laymen", but they showed considerably greater confidence in their predictions. In accordance with Griffin and Tversky's (1992) explanation of the overconfidence bias, the reason for this probably comes from the way the bettors use their information. For example, it has been shown that sport teams that play at home have a higher probability of winning the match than away teams (Nilsson \& Andersson, 2010). This means that the information about where the game is played has a fairly good predictive validity (i.e., weight). But, when predicting outcomes, expert bettors often rely more on their broad knowledge about each team (i.e., strength of evidence), such as the form of particular players or injuries within the team, which usually have smaller predictive validity (Andersson \& Ekman, 2003). Therefore, we should expect frequent, knowledgeable bettors to be more 
overconfident than less knowledgeable people who rarely or never bet. The latter have far less knowledge and evidence which they could base their confidence on.

\subsection{Conjunction fallacy}

According to Tversky and Kahneman (1983), "perhaps the simplest and the most basic qualitative law of probability is the conjunction rule: the probability of a conjunction, $\mathrm{P}(\mathrm{A} \& \mathrm{~B})$, cannot exceed the probabilities of its constituents, $\mathrm{P}(\mathrm{A})$ and $\mathrm{P}(\mathrm{B})$, because the extension (or the possibility set) of the conjunction is included in the extention of its constituents." (p. 1). However, in well-known Linda problem, a majority of respondents (85\%) declared the conjunction (Bank teller \& feminist) to be more probable than its less representative component (Bank teller), thus committing the conjunction fallacy.

One of the possible explanations of this phenomenon is the representativeness heuristic. According to this explanation, instead of making judgments about probabilities, people make conclusions about representativeness (Kahneman, 2002; Kahneman \& Frederick, 2002). Linda is more representative of a bank teller and a feminist than just a bank teller, and thus respondents estimate the probability of Linda being a bank teller and a feminist as greater than the probability of Linda being "only" a bank teller. However, not everybody agrees with this interpretation of the conjunction fallacy. For example, Costello (2009) believes that the conjunction fallacy does not necessary mean that the reasoning about conjunctions deviates from the standard probability theory, but that, due to certain amount of "noise" in the constituent probabilities, conjunction fallacy can occur even if the conjunction judgments are perfectly in line with the probability theory.

Another account of the conjunction fallacy is the "configural weighted average (CWA) hypothesis" (Nilsson, 2008; Nilsson, Winman, Juslin, \& Hansson, 2009). According to the CWA hypothesis, participants will assess the probability of the conjunction first by assessing the probability of each of the components in the conjunction, assigning them a weight and finally adding them. In other words, instead of multiplying the probabilities of components, participants are averaging them, inevitably making the conjunction fallacy. For example, the probability of the conjunction "Linda is a bank teller and is active in the feminist movement" will be assessed first by assigning greater weight to the less probable assertion (Linda is a bank teller) and lower weight to the more probable claim (Linda is active in the feminist movement), and then by aggregating these weighted claims. Thus, if a person estimates the probability of Linda being a bank teller to be 0.2 , and the probability that she is active in the feminist movement to be 0.8 , and then assigns weights of 0.7 to the less likely and 0.3 to the more likely claim, her integration of these information would proceed as follows:

$$
\mathrm{P}(\mathrm{B} \& \mathrm{~F})=0.7 \times 0.2+0.3 \times 0.8=0.38 \text {. }
$$

This person has committed a conjunction fallacy by assessing the probability of a conjunction (B\&F) to be higher than the probability of one component contained in the conjunction (B). The main difference between the CWA hypothesis and the representativeness heuristic explanation is that the representativeness heuristic attributes the error to the way in which the subjective probabilities are estimated while the CWA hypothesis attributes it to the way in which they are combined (Nilsson, 2008). Averaging, as a way of information integration, is especially useful when the components that comprise a conjunction are presented in a numerical form (Stolarz-Fantino, Fantino, Zizzo, \& Wen, 2003). Therefore, all eventual conjunction errors in betting could probably be best explained by the CWA hypothesis.

In fact, the conjunction fallacy in sports betting has only been rarely examined. Nilsson and Andersson (2010) conducted a study in which they presented their participants with various outcomes of football matches that varied in the likelihood of success (i.e., probability). When the conjunction consisted of the outcome with a low or intermediate probability that was combined with one or two outcomes that had high probability, it was perceived as more likely than the less likely outcome presented alone. This was not true when one outcome with a low or intermediate probability was combined with another outcome with a low likelihood of success. Thus, it would seem that bettors are not exempt from making the conjunction fallacy. However, generalizability of the Nilsson and Andersson's (2010) conclusions seems limited considering that the study was conducted on a small sample of individuals without specific references about their betting experiences (other than the participants on average betted 1.8 times per month).

\subsection{Influence of the task format on cognitive biases}

When predicting future events, people often disregard information about statistical distributions of an event's outcomes. In the case of betting on football matches, this information is the number of possible outcomes ("home win", "draw" or "away win"). Relying on distributional information in unpredictable environments, such as the outcomes of football matches, could increase the accuracy of prediction and reduce the overconfidence bias.

One way of forcing people to rely more heavily on distributional information is providing them with the frequency format of the task at hand (Brenner, Koehler, Liberman, \& Tversky, 1996; Kahneman, 2011). Tversky and Kahneman (1983) argued that formulating a task in terms of frequencies encourages subjects to recognize the fact that a claim (e.g., Linda is a bank teller) includes a conjunction (e.g., Linda is a bank teller and is active in the feminist movement). In order to test this hypothesis, they told their participants, the undergraduate students at Stanford University and UBC, that a health survey was conducted on a representative sample of adult males in British Colombia of all ages and occupations, and asked them to give the estimates that include two outcomes (i.e., conjunctions). In the case of frequency task, participants' task was to estimate "out of 100 people, how many have suffered one or more heart attacks" and "out of 100 people how many have suffered one or more 
heart attacks and are older than 55 years of age". In the probability format, their task was to "estimate the probability that the randomly selected person from the sample has suffered one or more heart attacks" and that "he has suffered one or more heart attacks and is older than 55 years of age." In the former case only $25 \%$ of subjects made the conjunction fallacy compared to probability-based task in which 65\% of subjects made the fallacy (Kahneman \& Tversky, 1996; Tversky \& Kahneman, 1983). The authors interpreted the results with the assumption that formulating the task in frequency terms leads to a spatial representation of the problem "which makes the relation of set inclusion particularly salient" (Kahneman \& Tversky, 1996, p. 586). Supporting evidences for the frequency format come also from other studies. Fiedler (1988) found that, in the frequency framework, $75-89 \%$ of people correctly followed the conjunction rule, the phenomenon that he attributed to the removal of the ambiguous term "probability" from the problem. Gigerenzer (1994) makes the case that the frequency representation is sufficient to make this cognitive illusion disappear. He claims that frequencies are an efficient representation of information because they mimic the way information was encountered throughout most of human evolution. In the case of a single event probability people are confused by the ambiguous reference class, contrary to the frequencies where the reference class is clarified (Gigerenzer, 2008). As our brain is predisposed to represent the information in frequencies and not probabilities, the biases should disappear when the information is given in the frequency format. Gigerenzer (1994) makes the case that violations of the probability laws in case of single events should not be labeled as errors or biases because the probability theory deals with the classes of events and not a single event.

However, results of studies exploring the influence of task format are far from unambiguous. Mellers, Hertwig, and Kahneman (2001) found that the frequency format eliminated the conjunction fallacy only in minority of presented cases; those without filler items and where "and are" and "who are" conjunction phrases were included (e.g. "of 100 people, how many are bank tellers and are feminists?"). Tentori, Bonini, and Osherson (2004) did not find a statistically significant difference in the magnitude of the bias between the frequency and probability format of the task, neither in Scandinavia nor in Volleyball problem. In the first problem subjects committed fallacy if they thought that the typical Scandinavian individual has blond hair and blue eyes instead of just blond hair, whereas in the second the fallacy was made if the typical female volleyball player was estimated to be less than 21 years old and taller than $1.77 \mathrm{~m}$ instead of just being younger than 21 years old. Participants made the same proportion of conjunction fallacies when answering the tasks formulated as frequencies as those given in the probability format. Wedell and Moro (2008) also failed to find a significant reduction in the conjunction bias due to the frequency effect across eight different tasks presented to the participants in two experiments. However, they did point out that although "shifting the focus from probabilities to frequencies did not significantly reduce conjunction errors in either Experiment 1 or 2, it trended in that direction" (Wedell \& Moro, 2008, p. 125). Thus, future research on the effect of task format to occurrence of cognitive biases is warranted.

\section{Aim and hypotheses}

The aim of our study was to contribute to a better understanding of the phenomenon of the overconfidence bias and the conjunction fallacy by examining their role in betting on sport outcomes. In our study we tried to identify how the prediction of football matches outcomes is influenced by the level of betting experience (frequent vs. sporadic bettors) and the task formats (probability vs. frequency).

Based on earlier research on overconfidence bias in low predictable environments (Andersson \& Ekman, 2003; Andersson et al., 2009; Torngren \& Montgomery, 2004) we expected that the frequent bettors will be significantly more confident about their evaluations than sporadic bettors even for predictions with the same level of accuracy (H1). Although there are explanations (e.g., the CWA hypothesis) why people are in general prone to the conjunction fallacy, those explanations tell us nothing about the possible differences in susceptibility to the conjunction fallacy between frequent and sporadic bettors. To the best of our knowledge, the relationship between the conjunction fallacy and betting experience has not been examined. However, it might be possible to draw some expectations based on the research about gambling, as gamblers are in many ways similar to bettors. Generally, irrational beliefs are more common among frequent gamblers than among people who are not prone to this type of behavior (Delfabbro, Lahn, \& Grabosky, 2006; Kallmen, Andersson, \& Andren, 2008). Frequent gamblers believe that their successes are the result of their gambling abilities, claim that they use their experience and knowledge extensively while gambling and view their losses as 'near misses' (the belief that the chances to win increase if one has been previously close to win; Kallmen et al., 2008, p. 442). If frequent bettors resemble gamblers, we should expect them to have more irrational beliefs and cognitive biases about betting than inexperienced bettors. Therefore, we believe that in addition to the general factors which make people more prone to the conjunction fallacy, additional characteristics of frequent bettors make them more prone to the irrational beliefs. Thus, we expected that the frequent bettors will be more susceptible to the conjunction fallacy than sporadic bettors ( $\mathrm{H} 2)$.

As stated earlier, the research about influence of frequency format on cognitive biases has been conflicting and inconclusive. However, following Gigerenzer's $(1994,2008)$ remarks about the influence of frequency format on cognitive biases, we expected that formulating the task in frequency format will reduce the susceptibility to overconfidence bias (H3). Also, if we carefully analyze the studies that do and do not find the effect of the task format on the conjunction fallacy, the occurrence of this type of bias could be related to type of response the subjects are required to provide. When subjects are required to give a frequency estimate instead of providing the rankings of options, it seems that the frequency format reduces the fallacy (Moro, 2009). Hertwig and Chase (1998) showed that people are very good in providing the estimates but very bad at providing the 
rankings. As the task in our study is to provide the estimate and not to rank the options, we expected that formulating the task in frequency format will reduce the susceptibility to conjunction fallacy (H4), compared to the probability task format.

\section{Method}

\subsection{Participants}

The groups of frequent and sporadic bettors were invited to participate in the study via social networks and e-mail. The researchers approached their friends and acquaintances for whom they knew that either regularly bet in the outcomes of football matches ("frequent bettors") or rarely/never engage in that kind of behavior ("sporadic bettors"). A total of 130 male subjects agreed to participate in the study, among whom 67 were considered to be frequent and 63 sporadic bettors. The responses to a question about frequency of betting behavior provided support to the recruitment procedure. Fourteen of our 67 frequent bettors bet every day, 31 bet few times a week, 16 once a week and six bet few times a month. As far as the sporadic bettors are concerned, 27 of them have never bet and 36 bet few times a year at most. The two groups of respondents differed also in self-reported knowledge about football. The majority of frequent bettors (93\%) considered themselves to have excellent or good knowledge of European football, while the percentage of the sporadic bettors who had the same estimate of football knowledge was much lower (32\%).

The frequent bettors were on average older than the sporadic bettors ( 30.2 vs. $26.2 ; t(128)=2.84, p=.006$ ) and the two groups differed significantly in educational structure $\left(\chi^{2}(1)=7.19, p=.004\right)$. Considerably more frequent bettors had secondary education compared to sporadic bettors $(40.3 \%$ vs. $17.5 \%)$ who in turn more often had college education than the frequent bettors ( $82.5 \%$ vs. $59.7 \%$ ). The differences in the educational and age structure between the two samples were taken into consideration in the analysis.

\subsection{Instrument and procedure}

We devised an online questionnaire that consisted of 120 items. Six items examined general information about the participants while the other 114 resembled betting tickets in which participants had to evaluate whether offered outcomes of football match(es) will occur. Out of 114 items, 74 listed outcomes of the single matches that took place in eight European leagues during the week when the research was conducted (Spanish, English, Italian, French, German, Belgian, Dutch and Russian premier football leagues). The remaining 40 items were conjunctions, pairs of football matches consisting of the previously evaluated singles.

The matches were assigned with one of possible outcomes (a home win, a draw or an away win), and the participants' task was to judge whether the assigned outcome (in case of singles) or the combination of two outcomes (in case of combinations) will actually occur. In accordance with Nilsson and Andersson (2010), who listed the statistics that $48 \%$ of matches ended with a home win (1), $28 \%$ with a draw (X) and $24 \%$ with away win (2), we randomly distributed the outcomes in the indicated ratios across the 74 singles. The participants were not familiar with the process of the outcomes assignment. The singles with assigned outcomes were combined in 40 conjunctions. Considering that we wanted to have both likely-likely (consisting of two very probable singles) and likely-unlikely conjunctions (one very probable and one less probable single), we had to sort out singles by their probability. For this purpose we used the coefficients assigned to the matches by an online betting service. In order to arrange singles from the most probable to the least probable we recomputed given coefficients into probabilities. For example, if the coefficient on some outcome was 2, that meant that the betting service estimated the probability of the match ending with that outcome to be $100 / 2=50 \%$. The 10 most probable and the 30 least probable singles were used to form the conjunctions. We decided to use this ratio because only 10 outcomes had the probability greater than $60 \%$ and could, therefore, be considered as probable. The probabilities of more probable singles were all greater than $60 \%$, whereas those of the less probably singles were all under 35\%. Out of the 40 conjunctions we had formed from this pool of singles, 30 likely-unlikely conjunctions were designed by randomly pairing the 30 less probable singles with one of 10 very probable singles. The remaining 10 likely-likely conjunctions were designed by randomly pairing up very probable singles. By comparing the probabilities assigned to the conjunctions with the probabilities assigned to the singles, we were able to determine the measure of the conjunction fallacy made by our participants.

In order to investigate the influence of the task format (probability/frequency), we designed two questionnaires that differed only in the way in which the participants were required to evaluate the outcomes. The items were identical in both questionnaires. A half of the participants in each group differing in betting experience provided their evaluations in terms of probability, and they were given the following instruction:

"You will be shown some football matches which will be played next weekend. The matches are already assigned with the outcomes $(1, \mathrm{X}$ or 2$)$. Imagine that every match with a corresponding outcome represents a bet you will place in a betting office. That is, that your bet consists of only that one match (outcome) and nothing else. Your task is to evaluate whether it will be a winning bet and to state the level of confidence regarding your evaluation (in percentages). If you think that it will be a winning bet, answer "Yes", and if you think it will not be a winning bet, answer "No". The level of confidence about your evaluation must be anywhere between 50\% and 100\%. 50\% means that you are not confident 
about your evaluation of the outcome, that is, that you're guessing, while $100 \%$ means that you're completely confident about your evaluation. Your level of confidence can be expressed by any number between 50\% and 100\%."

After this instruction, the participants evaluated the singles which appeared as follows:

Liverpool - Manchester United 2

Yes No

How confident are you about your answer? (Evaluate in percentages. The evaluation must be in the range between $50 \%$ and $100 \%$ )

After evaluating 74 singles, the participants made predictions about the conjunctions in the same way, except the instruction in which we emphasized that the bet will be considered as the winning only if both outcomes occur.

The other half of participants in each group gave their evaluation in the frequency terms. An example of the instruction and an item is shown below.

"Imagine that the match on which you are placing a bet will be played a 100 times, and that you bet on the same stated outcome every time. Your task is to evaluate how many, out of 100 bets, you will win, that is, how many times the match will end in a stated outcome. Liverpool - Manchester United 2 Out of those 100 cases, in how many cases would this be a winning ticket?"

After they gave their estimate for a bet and clicked "Next" for the next one, the participants could not go back to the previous page to change their responses.

The version of the questionnaire intended for the sporadic bettors contained an explanation of the concepts related to betting which were used in this research (in Appendix A), and those participants first evaluated eight exercise outcomes that were not included in the data analysis.

\section{Results}

\subsection{Accuracy and confidence of sport betting predictions depending on the betting frequency and the task format}

In situations where participants answered in probability terms, we checked the accuracy of each evaluation (i.e., what really happened on the matches) for each subject, and calculated the percentage of accurate evaluations per subject (i.e., accuracy index).

In situations where participants made their evaluations in frequency terms, before computing the average accuracy we recoded their answers so that they correspond to evaluations made in probability terms. If a participant predicted that an outcome would occur in less than 50 cases (for example, 35 out of 100), we considered that he believed that that an outcome would not occur. In this particular case his confidence estimate was considered to be $65 \%(100-35)$. If a participant predicted that an outcome would occur in over 50 cases (for example 65), we assumed that he believed that the outcome would occur, and that he was $65 \%$ confident about his answer.

The proportion of accurate answers and the average confidence were then computed in the same way as in the case of evaluating in terms of probability. The specific case represented the participants who reported that the outcome will happen in 50 of 100 cases. In the analysis their accuracy was coded as 0.5 . However, the average percentage of these responses per participant was only $8.92 \%$ which, we believe, could not have had any major influence on our results.

The results of betting accuracy depending on the betting frequency and the task format are shown in Table 1. Relatively low accuracy of predictions made by both frequent and sporadic bettors is in line with our assumption that outcomes of football matches are rather unpredictable.

Two-way ANOVA used for testing differences in accuracy has shown a significant main effect of both independent variables on the accuracy of participants (Betting frequency: $F(1,126)=6.28, p<.05$; Task format: $F(1,126)=54.47, p<.001$ ). ${ }^{1}$ The interaction term was insignificant $(F(1,126)=1.02 . p>.05)$. Generally, the frequent bettors were more accurate in their evaluations than the sporadic bettors, while evaluating the matches in frequency terms significantly increased the accuracy of both groups of participants compared to the evaluations given in terms of probability.

As we said, the outcomes of football matches are rather unpredictable. However, only participants' confidence in their evaluations reveals if they are aware of that fact. Both in the probability and the frequency format, the confidence of our participants in their estimates were relatively high ranging from $65.78 \%$ for the sporadic bettors in the probability condition to $73.77 \%$ for the frequent bettors in the probability condition. The two-way ANOVA showed a significant main effect of the betting frequency $(F(1,126)=8.76, p<.05)$ and significant interaction between the betting frequency and the task format $(F(1,126)=13.18, p<.001)$. The main effect of the task format was insignificant $(F(1,126)=0.06, p=.81)$. The simple effects

\footnotetext{
${ }^{1}$ Considering that the samples of frequent and sporadic bettors differed in the age and education composition, we also conducted all our analyses as ANCOVAs where age and education were used as covariates. Substantive conclusions were the same, thus we reported the more parsimonious ANOVA results throughout the manuscript.
} 
Table 1

Descriptive statistics of accuracy indices (\% of correct predictions) depending on the betting frequency and rating format.

\begin{tabular}{lllll}
\hline Type of task & Frequent bettors & & Sporadic bettors \\
\cline { 2 - 3 } & $\mathrm{M}$ & $\mathrm{SD}$ & $\mathrm{M}$ & 52.57 \\
\hline Probability & 56.99 & 6.14 & 63.12 & 9.80 \\
Frequency & 65.00 & 6.01 & 6.36 \\
\hline
\end{tabular}

of the task type showed that the estimates given in the frequency terms produced a significant decrease in confidence among the frequent bettors in comparison to the evaluations in the probability terms $(F(1,126)=5.91 ; p=.016)$. The sporadic bettors displayed the opposite trend: evaluating in frequency terms significantly increased their confidence about their evaluations $(F(1,126)=7.29 ; p=.008)$. Of course this was also reflected in the simple effect of the betting frequency: when they evaluated in the probability terms, the frequent bettors were considerably more confident about their answers than the sporadic bettors $(73.77 \%$ vs. $65.78 \% ; F(1,126)=21.62 ; p<.001)$. However, when the evaluations are given in the frequency terms, both frequent and sporadic bettors were equally confident about their answers $(69.67 \%$ vs. $70.48 \%$; $(F(1,126)=0.23 ; p=.635)$.The significant interaction between the frequency of betting and the task format is given in Fig. 1.

\subsection{Overconfidence bias depending on the betting frequency and the task format}

The index of overconfidence was obtained by subtracting the accuracy index from the participant's average confidence. For example, if a participant accurately predicted $50 \%$ of the proposed outcomes, but was on average $60 \%$ confident in his evaluations his rate of overconfidence was $10 \%$. The procedure of overconfidence calculation was identical for both probability and frequency evaluations.

From the data shown in Fig. 2 we can conclude that both groups of participants in both situations displayed a certain degree of overconfidence. However, the two-way ANOVA revealed a significant main effect of the task format $(F(1,126)=33.87, p<.001)$ and a significant interaction of the task format and the betting frequency $(F(1,126)=4.13$, $p<.05)$. The main effect of the betting frequency was insignificant $(F(1,126)=2.76 ; p=.102)$. The simple effects analysis showed that both sporadic and frequent bettors had significantly lower levels of overconfidence in the frequency task format in comparison to the probability tasks format (sporadic bettors: $F(1,126)=31.81, p<.001$; frequent bettors: $F(1,126)=6.96$, $p=.009$ ). Although there was a trend that the difference between the two groups of participants was larger in the probability in comparison to the frequency condition, the simple effects of the betting frequency indicated that the differences between the two groups in overconfidence level in probability $(F(1,126)=2.67, p=.105)$, as well as in frequency task condition $(F(1,126)=1.53, p=.218)$ were insignificant. This inconsistency between a significant interaction term and insignificant simple effects results from the fact that the two statistical procedures test for slightly different things (see Keppel \& Wickens, 2004). In sum, the significant interaction term indicated that the frequency task format reduced the overconfidence of the frequent bettors more than the overconfidence among sporadic bettors. But the differences between the groups within the conditions were relatively small and, therefore, insignificant.

\subsection{Conjunction fallacy in relation to the betting frequency and the task format}

In order to quantify the participants' susceptibility to the conjunction fallacy for both task formats, we converted the evaluations from the probability format to the frequency format. For example, if a participant said that he was $80 \%$ confident that

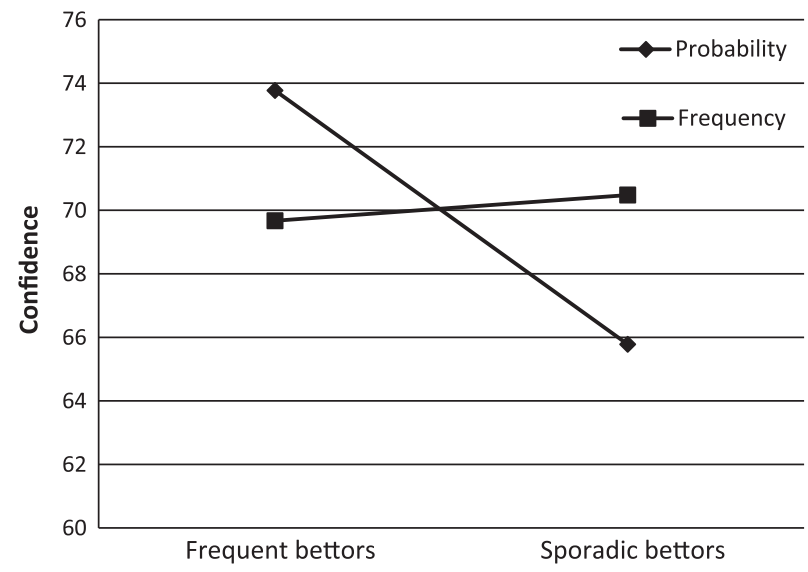

Fig. 1. Level of confidence in the evaluations of outcomes in relation to the betting frequency and the task format. 


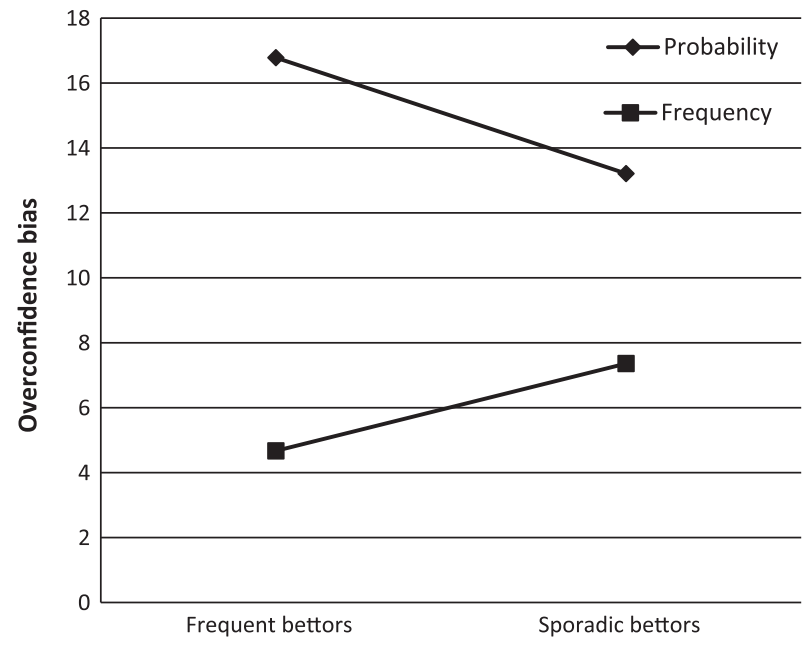

Fig. 2. Overconfidence bias in relation to the betting frequency and task format.

Table 2

Descriptive statistics of conjunction fallacy (\% of biases) depending on the betting frequency and the rating format.

\begin{tabular}{lllll}
\hline Type of task & \multicolumn{2}{l}{ Frequent bettors } & & \multicolumn{2}{l}{ Sporadic bettors } \\
\cline { 2 - 3 } & $\mathrm{M}$ & $\mathrm{SD}$ & $\mathrm{M}$ & \multicolumn{2}{c}{ SD } \\
\hline Probability & 49.24 & 15.26 & 43.10 & 17.67 \\
Frequency & 53.23 & 27.24 & 35.96 & 25.93 \\
\hline
\end{tabular}

a certain outcome will not happen, it was considered equal to saying that it will come true in 20 out of 100 hypothetical matches. If he said that the outcome will happen and was $80 \%$ confident about his answer, that meant that the outcome will come true in 80 out of 100 matches. A participant made the conjunction fallacy if he evaluated that the ticket containing, for example, "Everton - Arsenal 1", will be the winning ticket in 40 out of 100 cases, but the ticket containing "Everton - Arsenal 1; Barcelona - Getafe 1" will be the winning ticket in 65 out of 100 cases. We computed in how many, out of 40 possible cases, did each participant make conjunction fallacy. The results are expressed in percentages and listed in Table 2.

Two-way ANOVA used for testing differences in susceptibility to conjunction fallacy showed a significant main effect of the betting frequency $(F(1,126)=9.02, p<.05)$ but the main effect of the task format $(F(1,126)=0.16, p=.686)$ and the interaction term between the task format and betting frequency were insignificant $(F(1,126)=2.04, p=.155)$. As can be seen in Table 2, the frequent bettors made significantly more conjunction fallacies than the sporadic bettors. The task format did not influence the occurrence of the conjunction fallacy.

\section{Discussion}

In this study we examined the occurrence of the overconfidence bias and the conjunction fallacy in betting on football matches. We focused on the two issues that we believe were not sufficiently explored in earlier studies: the influence of frequency-based task format on the two biases in sport betting, and the differences in the conjunction fallacy between frequent and sporadic bettors.

Our study showed that the frequent bettors were generally more accurate in their evaluations of the outcomes of football matches than the sporadic bettors. However, the differences in the predictions confidence between the two groups depended on the task format. When giving their judgments in probability terms the frequent bettors showed a considerably greater level of confidence than the sporadic bettors, but there was no difference in the level of confidence between the two groups when the bets were given as frequencies. Generally, the frequent bettors were significantly more susceptible to the conjunction fallacy in comparison to the sporadic bettors, but there were no differences in the overconfidence bias between the two groups. The frequency-based task format has significantly increased the accuracy of both groups of participants, and resulted in a considerable reduction of the overconfidence bias in comparison to the evaluations made in the probability terms. Contrary to our assumption, the task format did not have a significant influence on the conjunction fallacy occurrence. Therefore, our hypotheses received a mixed support: we confirmed hypothesis $\mathrm{H} 2$ and $\mathrm{H} 3$, but failed to confirm $\mathrm{H} 1$ and $\mathrm{H} 4$.

This research brings some good news. The accuracy of one's evaluations can be increased and overconfidence reduced if the betting task is formulated in frequencies instead of probability terms. It could be that the bets in the frequency terms 
made both the frequent and sporadic bettors reject strategies used when making probability judgments and partly take the "outside view" instead the "inside view". Kahneman and Tversky (1982) stated that "people generally prefer the singular mode, in which they take an 'inside view' of the causal system that most immediately produces the outcome, over an 'outside view' which relates the case at hand to a sampling scheme"(p. 518). In this case the inside view refers to the knowledge or beliefs regarding the properties of the team being evaluated such as the team's form, the form of individual players, line-up or injuries. As opposed to this judging from the inside, when judging from the outside decision is made "considering some set or reference class of instances that the individual case belongs to" (Lagnado \& Sloman, 2004, p.159). Kahneman and Tversky (1996) believe that formulating the problem in frequency terms provides the subjects with additional hints that they should use statistical judgment which makes them take an outside perspective regarding the problem (Lagnado \& Sloman, 2004). Considering relatively modest predictive validity of the "inside view" information, a logical conclusion would be that taking an "outside view" will result in better evaluations. The fact that the frequency format resulted with significant improvement in the accuracy and significant reduction in the overconfidence bias in both groups probably supports this position. However, these explanations are fairly speculative and warrant further research.

In the present study, we also explored susceptibility to the conjunction fallacy between frequent and sporadic bettors. The obtained results show that the frequent bettors made the conjunction fallacy significantly more often than the sporadic bettors, and, in contrast to the overconfidence, the use of the frequency-based task format did not reduce this type of bias. There are several possible explanations for this finding. It is possible that frequent bettors represent a selected group, more susceptible to irrational beliefs than average people. An interesting finding from the present study pointed in that direction. We identified a significant, although weak correlation between the frequency of the overconfidence bias and the frequency of the conjunction fallacy $(r=.17 ; p<.05)$. This could indicate that certain individuals have predispositions that make them more susceptible to cognitive biases and irrational decision-making which is certainly something that requires further research. Of course, an alternative explanation for this correlation could be that one bias affects both variables (i.e., the correlation occurs because individuals who overestimate conjunctive probabilities are bound to commit more conjunction errors). The other possibility is that the greater susceptibility of the frequent bettors to the conjunction fallacy in our study was a consequence of question ordering in this research. Specifically, questions containing conjunctions came last, after evaluation of the singles. It is possible that, while completing the task, the frequent bettors became more tired than the sporadic bettors due to their use and detailed analysis of greater quantity of information in the process of evaluating singles and that conscious deliberation requires more effort (Kahneman, 2011). In other words, obtained results might reflect a difference in the task load between the two groups that occurred due to different strategies utilized while solving identical problems. This possibility should be tested in future research.

Unlike the overconfidence bias, the conjunction fallacy has proven to be resistant to the task format manipulation. This finding seems to be consistent with earlier studies which showed that conjunction fallacy is resistant to various attempts to reduce it, including financial motives (Stolarz-Fantino et al., 2003; Tversky \& Kahneman, 1983; Zizzo, 2005) and training in logical thinking (Zizzo, 2005). Even the betting paradigm (Sides, Osherson, Bonini, \& Viale, 2001) was not efficient in reducing this type of bias as subjects were still willing to put their money on the conclusion that the conjunction was more probable than its constituents. This resistance to various interventions and the constant over-estimation of the conjunctions are probably one of the causes of poor evaluations and financial losses among frequent bettors. The questions about what is the cause of the resistance of the conjunction fallacy and are there any ways of reducing it, in the case of bettors remain to be answered by further research.

But how can we improve decisions bettors and other people make and reduce the biases that frequent them? One interesting intervention suggested by Milkman, Chugh, and Bazerman (2009) is encouraging people to think about the reasons why their conclusions could be wrong. It seems that this intervention can make people think carefully about decisions and to try to take a different position regarding the problem they think about. This way people can more easily avoid becoming so-called motivated skeptics (Kunda, 1990; Molden \& Higgins, 2005; Taber \& Lodge, 2006), looking for all kinds of justifications for their decisions even when they are wrong and trying to protect their self-esteem and self-image when "bad" decisions are threatening them. In this respect bettors are not much more different from other decision makers. Gilovich (1983) showed that, even though they apply the same strategies in both successful and unsuccessful evaluations, bettors assume responsibility for successes but rationalize failures by attributing them to factors which are beyond their control. This is one of the strategies Tetlock (1999) identified that experts use to rationalize their bad moves, thereby failing to critically evaluate them and learn from their mistakes. It would be interesting to examine whether this simple intervention, where people think why their decision should be wrong, can help bettors recognize their mistakes in conjunction evaluations.

There are several limitations of our study and related suggestion for future research. We will mention the three that seem the most salient. The first one relates to the influence of the item format on the overconfidence bias occurrence. In this study we used both the half range and the full range format. When estimating in the probability terms participants were asked to state their confidence on the half range scale (50-100). At the same time, when answering in the frequency terms participants were estimating their confidence on the full range scale (1-100). Therefore, there is a possibility the observed reduction in overconfidence between the probability and frequency format could be attributed to the different evaluation formats. Although this is a possibility that should be pursued in future studies, previous research indicates that the influence of the half-range/full-range response format should operate in the other direction. Klayman et al. (2006) showed that the halfrange format usually produces smaller level of overconfidence than the full range format. Therefore if the half-range/ 
full-range response format influenced our findings, earlier studies show that influence should operate in opposite direction, making observed differences between frequency and probability estimates smaller than they really are.

Second, in the frequency task condition confidence in bets was measured indirectly. We have assumed that the response to the question "Out of those 100 cases, in how many times this would be a winning ticket?" reflect the confidence in the decision. Future studies should consider whether our findings are replicated when an additional, direct question about confidence (e.g., "How confident are you in your evaluation?") is included in the research design.

The observed difference in susceptibility to the conjunction fallacy between the frequent and sporadic bettors held even when we controlled for the differences in educational levels between the two groups. But, future research should test whether this difference could be related to education in statistics. Knoth and Benassi (2009) stated that there is a possibility that statistical sophistication could contribute to recognizing the correct rule for evaluating conjunctions, while Tversky and Kahneman (1983) showed that statistical sophistication truly enhances evaluations in accordance with the conjunction rule, although it does not eliminate the error entirely. This point to the conclusion that people do not know how to use the conjunction rule and go with their instinctive but wrong conclusion.

\section{Appendix A}

The explanation of concepts related to betting intended for laymen

Dear Sir/Madam, before you begin solving the questionnaire, we will explain some terms related to betting which will be used throughout the questionnaire.

When indicating matches (pairs), home team is always stated before the guest team. So, if it reads, for example, Arsenal - Manchester United, it means that the match is played at Arsenal's stadium, that is, that Arsenal is the home team and that Manchester United is the guest team. In the opposite case, if it reads Manchester United - Arsenal, it would mean that in this match Manchester United is the home team and Arsenal the guest team.

Every match has three possible outcomes: home win, a draw (no winner) and away win. These outcomes are indicated with the following signs:

1 signifies home win

$\mathrm{X}$ signifies a draw (no winner)

2 signifies away win

Using the example of Arsenal - Manchester United match, it would appear as following:

Arsenal - Manchester United $1 \rightarrow$ this match ended with a home win, in this case, Arsenal

Arsenal - Manchester United $X \rightarrow$ this match ended with a draw, that is, there was no winner

Arsenal - Manchester United $2 \rightarrow$ this match ended with away win, in this case, Manchester United

Pairs included on "tickets" paid in the betting offices are signified in the same way. In this research you will evaluate such tickets which will contain one or two matches. More details regarding your task can be found on the following page.

\section{References}

Alpert, W., \& Raiffa, H. (1982). A progress report on the training of probability assessors. In D. Kahneman, P. Slovic, \& A. Tversky (Eds.), Judgment under uncertainty: Heuristics and biases (pp. 294-305). New York: Cambridge University Press.

Andersson, P., Ekman, M. \& Edman, J. (2003). Forecasting the fast and frugal way: A study of performance and information - processing strategies of experts and non-experts when predicting the World Cup 2002 in soccer. SSE/EFI working paper series in business administration No 2003:9. Stockholm School of Economics, Stockholm.

Andersson, P., Memmert, D., \& Popowicz, E. (2009). Forecasting outcomes of the World Cup 2006: Performance and confidence of bettors and laypeople. Psychology of Sport and Exercise, 10, 116-123.

Belsky, G., \& Gilovich, T. (1999). Why smart people make big money mistakes - and how to correct them. Lessons from the new science of behavioural economics. New York: FIRESIDE.

Brenner, L. A., Koehler, D. J., Liberman, V., \& Tversky, A. (1996). Overconfidence in probability and frequency judgments: A critical examination. Organizational Behavior and Human Decision Processes, 65(3), 212-219.

Costello, F. J. (2009). How probability theory explains the conjunction fallacy. Journal of Behavioral Decision Making, 22, 213-234.

Delfabbro, P., Lahn, J., \& Grabosky, P. (2006). It's not what you know, but how you use it: Statistical knowledge and adolescent problem gambling. Journal of Gambling Studies, 22, 179-193.

Fiedler, K. (1988). The dependence of the conjunction fallacy on subtle linguistic factors. Psychological Research, 50, 123-129.

Gigerenzer, G. (2008). Rationality for mortals: How people cope with uncertainty. New York: Oxford University Press.

Gigerenzer, G. (1994). Why the distinction between single-event probabilities and frequencies is important for psychology (and vice versa). In G. Wright \& P. Ayton (Eds.), Subjective probability (pp. 129-161). New York: Wiley.

Gilovich, T. (1983). Biased evaluation and persistence in gambling. Journal of Personality and Social Psychology, 44, 1110-1126.

Griffin, D., \& Tversky, A. (1992). The weighting of evidence and the determinants of confidence. Cognitive Psychology, 24, 411-435.

Hansson, P. (2007). A naïve sampling model of intuitive confidence intervals. (Doctoral dissertation). Department of Psychology, Umeå University, S-901 87 Umeå, Sweden. Retrieved from umu.diva-portal.org/smash/get/diva2:140742/FULLTEXT01.

Hertwig, R., \& Chase, V. (1998). Many reasons of just one: How response mode affects reasoning in the conjunction problem. Thinking and Reasoning, 4(4), 319-352. 
Hertwig, R., \& Gigerenzer, G. (1999). The “conjunction fallacy” revisited: How intelligent inferences look like reasoning errors. Journal of Behavioral Decision Making, 12, 275-305.

Kahneman, D. (2002). A perspective on judgment and choice: Mapping bounded rationality. American Psychologist, 58, 697-720.

Kahneman, D. (2011). Thinking, fast and slow. New York: Farrar, Straus and Giraux.

Kahneman, D., \& Frederick, S. (2002). Representativeness revisited: Attribute substitution in intuitive judgment. In T. D. Gillovich, D. Griffin, \& D. Kahneman (Eds.), Heuristics and biases: Psychology of Intuitive Judgment (pp. 49-81). Cambridge: Cambridge University Press.

Kahneman, D., \& Tversky, A. (1996). On the reality of cognitive illusions. Psychological Review, 103, $582-591$.

Kahneman, D., \& Tversky, A. (1982). Variants of uncertainty. In D. Kahneman, P. Slovic, \& A. Tversky (Eds.), Judgment under uncertainty: Heuristics and biases. Cambridge: Cambridge University Press.

Kallmen, H., Andersson, P., \& Andren, A. (2008). Are irrational beliefs and depressive mood more common among problem gamblers than non-gamblers? A survey study of Swedish problem gamblers and controls. Journal of Gambling Studies, 24(4), 441-450.

Keppel, G., \& Wickens, T. D. (2004). Design and analysis: A researcher's handbook. Pearson.

Klayman, J., Soll, J. B., Juslin, P., \& Winman, A. (2006). Subjective confidence and the sampling of knowledge. In K. Fiedler \& P. Juslin (Eds.), In the beginning there is a sample: Information sampling as a key to understanding adaptive cognition. Cambridge, UK: Cambridge University Press.

Klein, G., \& Kahneman, D. (2009). Conditions for intuitive expertise: A failure to disagree. American Psychologist, 64(6), 515-526.

Knoth, R. L., \& Benassi, V. A. (2009). Measurement of the conjunction error in social judgment: Answer choice and answer justification. Social Behavior and Personality, 37, 481-490.

Kunda, Z. (1990). The case for motivated thinking. Psychological Bulletin by the APA, 108, 480-498.

Lagnado, D. A., \& Sloman, S. A. (2004). Inside and outside probability judgment. In D. Koehler \& N. Harvey (Eds.), Blackwell handbook of judgment and decision making. Oxford: Blackwell Publishing Ltd.

Mellers, B., Hertwig, R., \& Kahneman, D. (2001). Do frequency representations eliminate conjunction effects? An exercise in adversarial collaboration. Psychological Science, 12, 269-275.

Milkman, K. L., Chugh, D., \& Bazerman, M. H. (2009). How can decision making be improved? A Journal of the Association for Psychological Science, 4, $379-383$.

Molden, D. C., \& Higgins, E. T. (2005). Motivated thinking. In K. J. Holyoak \& R. G. Morrison (Eds.), The Cambridge handbook of thinking and reasoning. New York: Cambridge university press.

Montier, J. (2007). Behavioral investing: A practitioner's guide to applying behavioural finance. Chichester: John Wiley \& Sons Ltd..

Moro, R. (2009). On the nature of conjunction fallacy. Synthese, 171(1), 1-24.

Nilsson, H. (2008). Exploring the conjunction fallacy within a category learning framework. Journal of Behavioral Decision Making, 21, 471-490.

Nilsson, H., \& Andersson, P. (2010). Making the seemingly impossible appear possible. Effects of conjunction fallacies in evaluation of bets on football games. Journal of Economic Psychology, 31, 172-180.

Nilsson, H., Winman, A., Juslin, P., \& Hansson, G. (2009). Linda is not a bearded lady: configural weighting and adding as the cause of extension errors. Journal of Experimental Psychology, 138, 517-534.

Sides, A., Osherson, D., Bonini, N., \& Viale, R. (2001). On the reality of conjunction fallacy. Memory and Cognition, 30, 191-198.

Stolarz-Fantino, S., Fantino, E., Zizzo, D. J., \& Wen, J. (2003). The conjunction effect: New evidence for robustness. The American Journal of Psychology, 116(1), $15-34$.

Svenson, O. (1981). Are we all less risky and more skillful than our fellow drivers? Acta Psychologica, 47, $143-148$.

Taber, C. S., \& Lodge, M. (2006). Motivated skepticism in the evaluation of political beliefs. American Journal of Political Science, 50, 755-769.

Tentori, K., Bonini, N., \& Osherson, D. (2004). The conjunction fallacy: A misunderstanding about conjunction? Cognitive Science, $28,467-477$.

Tetlock, P. E. (1999). Theory - driven reasoning about plausible pasts and probable future in world politics: are we prisoners of our preconceprions? American Journal of Political Science, 43, 335-366.

Torngren, G., \& Montgomery, H. (2004). Worse than chance? Performance and confidence among professionals and laypeople in the stock market. Behavioural Finance, 5(3), 148-153.

Tversky, A., \& Kahneman, D. (1983). Extensional versus intuitive reasoning: The conjunction fallacy in probability judgment. Psychological Review, 90, $293-315$.

Vallone, R. P., Griffin, D. W., Lin, S., \& Ross, L. (1990). Overconfident prediction of future actions and outcomes by self and others. Journal of Personality and Social Psychology, 58(4), 582-592.

Wedell, D. H., \& Moro, R. (2008). Testing boundary conditions for the conjunction fallacy: Effects of response mode, conceptual focus and problem type. Cognition, 107, 105-136.

Zizzo, D. J. (2005). Simple and compound lotteries: Experimental evidence and neural network modelling. In D. J. Zizzo (Ed.), Transfer of knowledge in economic decision-making (pp. 166-193). Palgrave Macmillan. 